Research Paper

\title{
Longitudinal Associations between Triglycerides and Metabolic Syndrome Components in a Beijing Adult Population, 2007-2012
}

\author{
Li-Xin Tao ${ }^{1,2}$, Kun Yang 1,2, Xiang-Tong Liu'1,2, Kai Cao1,2, Hui-Ping Zhu1,2, Yan-Xia Luo ${ }^{1,2}$, Jin Guo ${ }^{1,2}$, \\ Li-Juan $\mathrm{Wu}^{1,2}$, Xia Li ${ }^{1,2,3 凶}$, Xiu-Hua Guo ${ }^{1,2 \bowtie}$ \\ 1. School of Public Health, Capital Medical University, Beijing 100069, China \\ 2. Municipal Key Laboratory of Clinical Epidemiology, Capital Medical University, Beijing 100069, China \\ 3. Department of Epidemiology \& Public Health, University College Cork, Cork 78746, Ireland
}

$\triangle$ Corresponding authors: Prof. Xiu-Hua Guo, School of Public Health, Capital Medical University. Beijing 100069, China. Tel: +861083911508; fax: +861083911508; E-mail: statguo@ccmu.edu.cn. Xia Li, Department of Epidemiology \& Public Health, University College Cork, Cork 78746, Ireland. Tel: +861083911778; fax: +861083911778; E-mail: lixia_new@163.com.

(C) Ivyspring International Publisher. Reproduction is permitted for personal, noncommercial use, provided that the article is in whole, unmodified, and properly cited. See http://ivyspring.com/terms for terms and conditions.

Received: 2015.10.28; Accepted: 2016.05.18; Published: 2016.06.01

\begin{abstract}
Background: Longitudinal associations between triglycerides (TG) and other metabolic syndrome (MetS) components have rarely been reported. The purpose was to investigate the longitudinal association between TG and other MetS components with time.

Methods: The longitudinal study was established in 2007 on individuals who attended health check-ups at Beijing Tongren Hospital and Beijing Xiaotangshan Hospital. Data used in this study was based on $\mathbf{7 4 8 9}$ participants who had at least three health check-ups over a period of 5-year follow up. Joint model was used to explore longitudinal associations between TG and other MetS components after adjusted for age.

Results: There were positive correlations between TG and other MetS components except for high density lipoprotein (HDL), and the correlations increased with time. A negative correlation was displayed between TG and HDL, and the correlation also increased with time. Among all five pairs of TG and other MetS components, the marginal correlation between TG and body mass index (BMI) was the largest for both men and women. The marginal correlation between TG and fasting plasma glucose was the smallest for men, while the marginal correlation between TG and diastolic blood pressure was the smallest for women.

Conclusions: The longitudinal association between TG and other MetS components increased with time. Among five pairs of TG and other MetS components, the longitudinal correlation between TG and BMI was the largest. It is important to closely monitor subjects with high levels of TG and BMI in health check-up population especially for women, because these two components are closely associated with development of hypertension, diabetes, cardiovascular disease and other metabolic diseases.
\end{abstract}

Key words: Triglycerides, Metabolic syndrome components, Longitudinal correlation, Joint model

\section{Introduction}

Metabolic syndrome (MetS) refers to a group of inter-related risk factors that include hyperglycemia, elevated blood pressure (BP), elevated triglycerides (TG), low high-density lipoprotein (HDL) levels, and obesity (in particular, central obesity) [1]. MetS is a strong predictor of cardiovascular disease (CVD), diabetes, stroke, and all-cause mortality, and is becoming a major public-health challenge worldwide [2-6]. Although, it is not yet clear whether MetS has a single cause, it is acknowledged that the most critical pathophysiology is insulin resistance $[7,8]$, and therefore the importance of central obesity was 
established [9].

Hypertriglyceridemia is often observed in subjects with MetS, type 2 diabetes, stroke, or combined with hyperlipidemia $[10,11]$. The level of TG is an independent risk factor for CVD events, independent of serum HDL or low-density lipoprotein (LDL) levels [12]. A number of meta-analyses have been published demonstrating associations between TG levels and CVD risk, independent of HDL level [13, 14].

There are several cross-sectional studies regarding the association between TG and other MetS components. In a cross sectional survey study on 266 Turkish elderly aged people, TG was reported to be significantly correlated with weight, body mass index (BMI), waist circumference (WC), waist/hip and waist/height [15]. In another study involving healthy Japanese women, high level of TG was also associated with increased level of BMI [16]. Other two studies have also recognized positive correlation between TG and other metabolic risk factors using partial correlation analysis and pearson correlation analysis $[17,18]$. However, there have been limited data about longitudinal associations between TG and other MetS components. To fully understand the longitudinal association between TG and other MetS components, longitudinal studies are required, especially using repeated measures of MetS components.

Therefore, the aim of the present study was to assess longitudinal associations between TG and other MetS components in a Beijing adult population from 2007 to 2012.

\section{Methods}

\section{Participants}

The longitudinal study was set up in 2007 on adults who attended health check-ups at Beijing Tongren Hospital and Beijing Xiaotangshan Hospital. A total of 7489 subjects with at least three health check-ups in the 5-year follow-up were enrolled in the study. Individuals with a previous diagnosis of CVD, cerebral infarction, gastric cancer, or those who had undergone coronary artery bypass surgery, coronary stenting surgery or gastrectomy, or those who had MetS, obesity, dyslipidemia, hyperglycemia, or hypertension at baseline were excluded. The study was approved by the ethics committee of Capital Medical University of China, and performed in accordance with the principles of Declaration of Helsinki (2013SY26). All participants gave their informed written consents.

\section{Definition of MetS}

MetS was diagnosed if participants had three or more of the following risk determinants according to the Joint Interim Statement criteria [1]. However, WC was not measured because of limited health check-up site, and BMI was taken as a substitute for the component of obesity [19]. The determinants were as follows:

- Obesity: $\mathrm{BMI} \geq 28 \mathrm{~kg} / \mathrm{m}^{2}$;

- Elevated TG (drug treatment for elevated TG is an alternate indicator) $\geq 150 \mathrm{mg} / \mathrm{dL} \quad(1.7$ $\mathrm{mmol} / \mathrm{L}$ );

- Reduced HDL (drug treatment for reduced HDL is an alternate indicator) $<40 \mathrm{mg} / \mathrm{dL}(1.0$ $\mathrm{mmol} / \mathrm{L})$ in males, $<50 \mathrm{mg} / \mathrm{dL}(1.3 \mathrm{mmol} / \mathrm{L})$ in females;

- Elevated BP (antihypertensive drug treatment in a patient with a history of hypertension is an alternate indicator) systolic blood pressure (SBP) $\geq 130 \mathrm{mmHg}$ and/or diastolic blood pressure (DBP) $\geq 85 \mathrm{mmHg}$;

- Elevated fasting plasma glucose (FPG) (drug treatment of elevated glucose is an alternate indicator) $\geq 100 \mathrm{mg} / \mathrm{dL}$.

\section{Measurements}

The participants underwent routine physical examinations that included the measurement of height, weight, BP, and overnight fasting blood sampling. Weight and height were measured without shoes, and BMI was calculated as weight $(\mathrm{kg})$ divided by squared height $(\mathrm{m})$. The measurement of weight was undergone at least twice for the improvement of reliability. The intra variability of weight was $<5 \%$. BP was measured on the right upper arm and maintained at the level of the heart with participants in sitting position. BP was measured by trained and certified nurses working in Beijing Tongren Hospital and Beijing Xiaotangshan Hospital. Before measuring $\mathrm{BP}$, subjects were at rest for at least 5 minutes. The trained nurse will measure BP three times in the following 30 minutes. During the $30 \mathrm{~min}$ preceding the measurements, the subjects were required to refrain from smoking or consuming caffeine. A standard mercury sphygmomanometer was used with 1 of 4 cuff sizes (pediatric, regular adult, large adult, or thigh) based on the participant's arm circumference. Three readings of SBP and DBP were recorded, and the average of the last two measurements was used for data analysis.

Blood samples were obtained from antecubital vein into tubes containing ethylenediaminetetraacetic acid (EDTA) in the morning after an overnight fasting period. Then the samples were stored in $4^{\circ} \mathrm{C}$ refrigerator, and they will be analyzed in 8 hours. HDL, TG, and FPG were measured by enzymatic method using a chemistry analyzer (Beckman LX 20, America) at Department of Clinical Laboratory in 
Beijing Tongren Hospital and Beijing Xiaotangshan Hospital. All analyses performed in accordance with the manufacturer's recommendations.

\section{Statistical Analysis}

\section{Missing Data Imputation and Data analysis}

To account for missing values, multiple imputation (MI) was performed. Because the imputation method of choice depended on the pattern of missing data and the type of the imputed variables, the Markov Chain Monte Carlo method was chosen to avoid loss of generality. The MI procedure of SAS software package (version 9.2; SAS Institute, Chicago, IL, USA) was used [20].

Data were expressed as mean \pm standard deviation or, for non-normally distributed variables, as median and interquartile range. To compare the differences between groups, student's $t$ test or Wilcoxon rank sum test was used to determine differences between groups. Joint model was conducted using SAS mixed procedure to account for longitudinal correlations between TG and other MetS components. Data were analyzed using SAS software package and $P<0.05$ was considered as significant.

When there were two continuous response variables, $Y_{1}$ and $Y_{2}$, they were measured over time for a number of subjects. Each of the variables was described using the linear mixed-effects model:

$$
\begin{aligned}
& Y_{1 \mathrm{i}}(t)=\mu_{1}(t)+a_{1 \mathrm{i}}+b_{1 i} t+\varepsilon_{1 i}(t) \\
& Y_{2 \mathrm{i}}(t)=\mu_{2}(t)+a_{2 i}+b_{2 i} t+\varepsilon_{2 i}(t)
\end{aligned}
$$

The marginal correlation between $Y_{1}$ and $Y_{2}$ at time $t$ is given by:

$$
r_{M}(t)=\frac{\operatorname{Cov}\left(Y_{1 i}(t), Y_{2 i}(t)\right)}{\sqrt{\operatorname{Var}\left(Y_{1 i}(t)\right) \times \operatorname{Var}\left(Y_{2 i}(t)\right)}}=\frac{\sigma_{a_{1} a_{2}}+t \sigma_{a_{1} b_{2}}+t \sigma_{a_{2} b_{1}}+t^{2} \sigma_{b_{1} b_{2}}}{\sqrt{\left(\sigma_{a_{1}}^{2}+2 t \sigma_{a_{1} b_{1}}+t^{2} \sigma_{b_{1}}^{2}+\sigma_{1}^{2}\right) \times\left(\sigma_{a_{2}}^{2}+2 t \sigma_{a_{2} b_{2}}+t^{2} \sigma_{b_{2}}^{2}+\sigma_{2}^{2}\right)}}
$$

\begin{tabular}{|c|c|c|c|c|c|}
\hline Year & Variables & Men & Women & $t$ & $P$ \\
\hline \multirow[t]{8}{*}{2007} & $N$ & 3389 & 4100 & - & - \\
\hline & age & $41.55 \pm 10.81$ & $41.03 \pm 10.24$ & -2.13 & 0.0332 \\
\hline & BMI $\left(\mathrm{kg} / \mathrm{m}^{2}\right)$ & $23.42 \pm 2.50$ & $21.80 \pm 2.53$ & -27.88 & $<0.0001$ \\
\hline & FPG (mmol/L) & $4.88 \pm 0.42$ & $4.86 \pm 0.40$ & -2.12 & 0.0341 \\
\hline & $\mathrm{HDL}(\mathrm{mmol} / \mathrm{L})$ & $1.35 \pm 0.27$ & $1.63 \pm 0.30$ & 43.27 & $<0.0001$ \\
\hline & $\mathrm{SBP}(\mathrm{mmHg})$ & $111.25 \pm 9.11$ & $105.94 \pm 10.43$ & -23.49 & $<0.0001$ \\
\hline & DBP (mmHg) & $73.72 \pm 6.58$ & $69.87 \pm 7.42$ & -23.76 & $<0.0001$ \\
\hline & TG (mmol/L) & $1.04 \pm 0.35$ & $0.85 \pm 0.34$ & -24.62 & $<0.0001$ \\
\hline \multirow[t]{7}{*}{2012} & $N$ & 1744 & 2171 & - & - \\
\hline & BMI $\left(\mathrm{kg} / \mathrm{m}^{2}\right)$ & $24.26 \pm 2.70$ & $22.45 \pm 2.81$ & -20.36 & $<0.0001$ \\
\hline & FPG (mmol/L) & $5.16 \pm 0.53$ & $5.05 \pm 0.45$ & -6.94 & $<0.0001$ \\
\hline & $\mathrm{HDL}(\mathrm{mmol} / \mathrm{L})$ & $1.27 \pm 0.28$ & $1.57 \pm 0.33$ & 30.61 & $<0.0001$ \\
\hline & $\mathrm{SBP}(\mathrm{mmHg})$ & $119.58 \pm 13.32$ & $113.24 \pm 14.33$ & -14.31 & $<0.0001$ \\
\hline & $\mathrm{DBP}(\mathrm{mmHg})$ & $73.03 \pm 9.80$ & $69.56 \pm 9.71$ & -11.08 & $<0.0001$ \\
\hline & TG $(\mathrm{mmol} / \mathrm{L})$ & $1.33 \pm 0.74$ & $1.04 \pm 0.53$ & -14.06 & $<0.0001$ \\
\hline
\end{tabular}

Table 1. Basic characteristics and MetS components of subjects in 2007 and 2012

Table 2. Fixed-Effects Tests of age for all subjects

\begin{tabular}{llllll}
\hline Pairs of MetS components Outcome & \multicolumn{3}{l}{ Men } & \multicolumn{3}{l}{ Women } \\
\cline { 2 - 6 } & & $t^{\#}$ & $p^{\#}$ & $t^{\#}$ & $p^{\#}$ \\
\hline TG and BMI & TG & 7.73 & $<0.0001$ & 20.79 & $<0.0001$ \\
& BMI & 7.62 & $<0.0001$ & 24.28 & $<0.0001$ \\
TG and FPG & TG & 7.30 & $<0.0001$ & 20.70 & $<0.0001$ \\
& FPG & 6.40 & $<0.0001$ & 3.93 & $<0.0001$ \\
TG and HDL & TG & 8.46 & $<0.0001$ & 22.17 & $<0.0001$ \\
& HDL & 1.89 & 0.0582 & -2.72 & 0.0066 \\
TG and SBP & TG & 7.43 & $<0.0001$ & 17.07 & $<0.0001$ \\
& SBP & 11.55 & $<0.0001$ & 24.20 & $<0.0001$ \\
TG and DBP & DBP & 13.80 & $<0.0001$ & 21.46 & $<0.0001$ \\
& TG & 7.23 & $<0.0001$ & 20.73 & $<0.0001$ \\
\hline
\end{tabular}

Abbreviations: MetS = metabolic syndrome; $\mathrm{BMI}=$ body mass index $; \mathrm{FPG}=$ fasting plasma glucose; $\mathrm{HDL}=$ high-density lipoprotein; $\mathrm{SBP}=$ systolic blood pressure; $\mathrm{DBP}=$ diastolic blood pressure; $\mathrm{TG}=$ triglycerides

\#: Joint Model was used.

\section{Results}

\section{Basic characteristics and MetS components of the subjects}

The basic characteristics and MetS components of the study population in 2007 and 2012 were shown in Table 1. There were 7489 individuals included in the study in 2007 at baseline (3389 men, $41.55 \pm 10.81$ years; 4100 women, $41.03 \pm 10.24$ years). Significant differences were displayed in the levels of age, BMI, FPG, HDL, SBP, DBP, and TG between men and women.

\section{Results of Joint Model}

Fixed-effects tests for age are displayed in Table 2. It was indicated that age had significant effects on the development of TG, BMI, FPG, SBP and DBP for men. And age had significant effects on the development of all MetS components for women.

The marginal correlations between TG and other MetS components after adjusted for age are presented in Table 3, Figure 1 and Figure 2.

Longitudinal correlations between TG and other MetS components for men adjusted for age were presented in Figure 1 . There were positive correlations between TG and other MetS components except for HDL, and the correlations gradually increased with time. A negative correlation was displayed between TG and HDL, and the correlation also increased with time. Among all five pairs of TG and other MetS components, the marginal correlation 
between TG and BMI was the largest, and the marginal correlation between TG and FPG was the smallest.

Longitudinal correlations between TG and other MetS components for women adjusted for age were displayed in Figure 2. Positive associations were detected between TG and other MetS components except for HDL, and the correlations increased rapidly with time. TG was negatively correlated with HDL, and the correlation also increased with time. Among all the five cases, the marginal correlation between TG and BMI was the largest, and the marginal correlation between TG and DBP was the smallest.

Table 3. The marginal correlation between TG and other MetS components for all subjects

\begin{tabular}{|c|c|c|c|c|c|c|c|}
\hline & $\begin{array}{l}\text { Pairs of MetS } \\
\text { components }\end{array}$ & 2007 & 2008 & 2009 & 2010 & 2011 & 2012 \\
\hline \multirow[t]{5}{*}{ Men } & TG and $\mathrm{BMI}^{*}$ & 0.2423 & 0.2433 & 0.2571 & 0.2792 & 0.3060 & 0.3350 \\
\hline & TG and $\mathrm{FPG}^{*}$ & 0.0654 & 0.0649 & 0.0777 & 0.0980 & 0.1213 & 0.1450 \\
\hline & TG and HDL ${ }^{*}$ & -0.1583 & -0.1682 & -0.1891 & -0.2173 & -0.2500 & -0.2848 \\
\hline & TG and $\mathrm{SBP}^{*}$ & 0.0830 & 0.0973 & 0.1170 & 0.1391 & 0.1616 & 0.1832 \\
\hline & TG and $\mathrm{DBP}^{*}$ & 0.0959 & 0.1092 & 0.1282 & 0.1495 & 0.1710 & 0.1915 \\
\hline \multirow[t]{5}{*}{ Women } & TG and $\mathrm{BMI}^{*}$ & 0.2140 & 0.3919 & 0.4793 & 0.5235 & 0.5479 & 0.5624 \\
\hline & TG and $\mathrm{FPG}^{*}$ & 0.0696 & 0.1518 & 0.1967 & 0.2201 & 0.2331 & 0.2409 \\
\hline & TG and $\mathrm{HDL}^{*}$ & -0.1708 & -0.3429 & -0.4312 & -0.4765 & -0.5016 & -0.5166 \\
\hline & TG and SBP* & 0.0927 & 0.2218 & 0.3000 & 0.3426 & 0.3668 & 0.3814 \\
\hline & TG and $\mathrm{DBP}^{*}$ & 0.0818 & 0.0909 & 0.1051 & 0.1227 & 0.1421 & 0.1621 \\
\hline
\end{tabular}

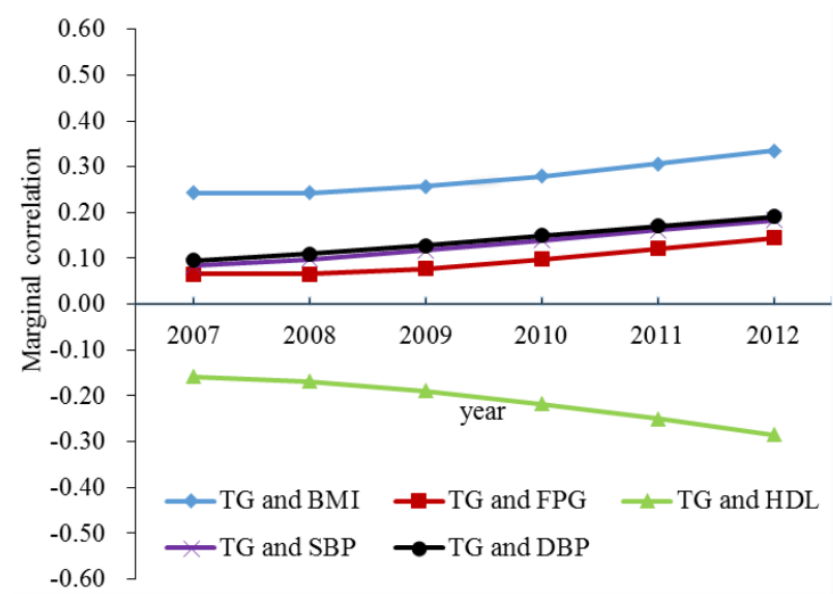

Figure 1. The marginal correlations between TG and other MetS components for men. Abbreviations: $\mathrm{BMI}=$ body mass index; $\mathrm{FPG}=$ fasting plasma glucose; $\mathrm{HDL}=$ high-density lipoprotein; $\mathrm{SBP}=$ systolic blood pressure; $\mathrm{DBP}=$ diastolic blood pressure; $\mathrm{TG}=$ triglycerides; MetS = metabolic syndrome.

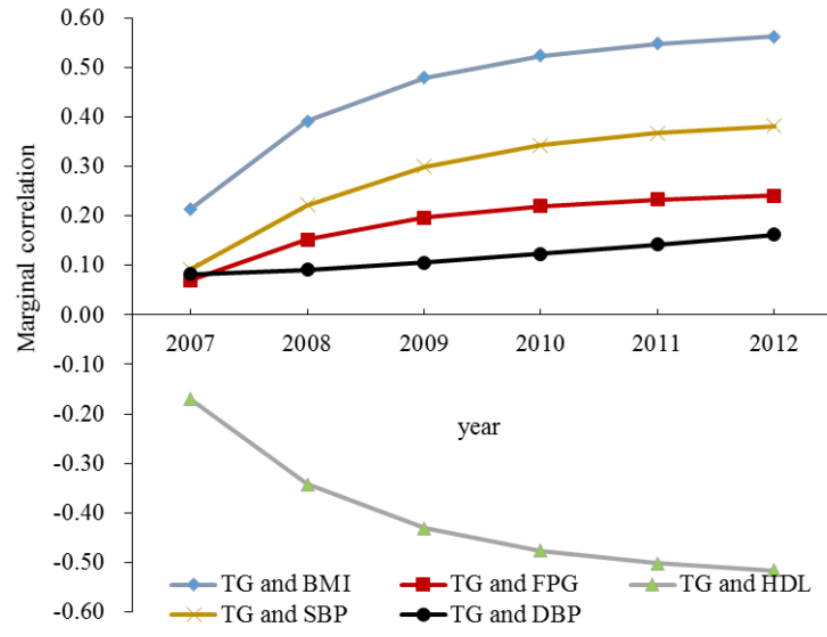

Figure 2. The marginal correlations between TG and other MetS components for women. Abbreviations: $\mathrm{BMI}=$ body mass index; $\mathrm{FPG}=$ fasting plasma glucose; $\mathrm{HDL}=$ high-density lipoprotein; $S B P=$ systolic blood pressure; $\mathrm{DBP}=$ diastolic blood pressure; $\mathrm{TG}=$ triglycerides; MetS = metabolic syndrome.

\section{Discussion}

Longitudinal associations between TG and other MetS components over time have rarely been considered in previous studies. Joint model is a novel method detecting longitudinal association between two outcomes.

Joint modeling two MetS components, rather than modeling them independently, and therefore incorporates additional information into the model that may increase estimation and prediction accuracy. Joint model has often been applied for longitudinal measurements and time-to-event data [21, 22]. The model also has been used for two longitudinal continuous measurements. One previous study using the joint model detected a very strong association between the development of two hearing thresholds and a slowly increasing change in the association between these thresholds over time [23]. However, there are few inferences about using the joint model to analyze the longitudinal association between TG and other MetS components with time.

In the present study, longitudinal associations between TG and other MetS components were assessed after adjusted for age. It was suggested that the marginal correlation between TG and BMI was the largest and the correlation increased with time for both men and women. And the correlation between TG and BMI is larger for women than men. The positive association was also reported in previous literature using partial correlation analysis in a cross sectional study [17]. But no inference has shown the longitudinal association between TG and BMI. Positive association between TG and BMI was also 
detected in the present study, as shown previously $[19,24]$. Therefore, it is important to closely monitor subjects with high levels of TG and BMI especially for women, because these two components are closely associated with development of hypertension, diabetes, CVD and other chronic diseases.

The marginal correlation between TG and FPG was positive and increased with time for men and women. But the correlation between TG and FPG is larger for women than men. Previous literatures have suggested that individuals with elevated FPG usually have a number of other metabolic abnormalities, including elevated TG, and elevated BP [24-26]. It has been reported that lipotoxicity by deposition of TG may be associated with beta-cell dysfunction and insulin resistance [27].

The marginal correlation between TG and HDL increased with time for both genders. The correlation between TG and HDL was extremely larger for men than women. The strong association between TG and HDL were consistent with previous results $[25,28$, 29]. One study has shown that an increase in TG level is commonly associated with a reduction in HDL level [30]. It has been indicated that hypertriglyceridemia stimulates the enzymatic activity of cholesterol ester transfer protein, which facilitates the transfer of TG from TG-rich lipoproteins to HDL and LDL in exchange for cholesteryl esters [31]. This leads to an increase in TG content of HDL and LDL.

The marginal correlation between TG and BP increased slowly with time for men and women in the present study. The correlation between TG and SBP was a little larger for women than men. But the correlation between TG and DBP for men was slightly larger for men than women. One previous study has shown a significant increase in plasma TG level as BP levels increased from optimally normal, across high-normal to hypertensive levels [32].

The strength of this research was that it was a longitudinal study of 5 years in a Beijing adult population. The present study assessed longitudinal association between TG and other MetS components for the first time. The limitation was the lack of WC as an indicator for central obesity, however, BMI was used for substitute. One study showed that the two measures of BMI and WC are closely correlated [33]. Another study indicated that most individuals with an abnormal BMI also have an abnormal WC. Both indexes of excess adiposity are positively associated with SBP, FPG, and TG and inversely associated with HDL [34]. One study based on a cross-sectional field survey targeted adults in China demonstrated that dyslipidemia, particularly hypertriglyceridemia and low HDL is associated with central obesity (measured with WC) using multivariate logistic regression [35].
A previous study used both BMI and WC has shown that either of the two measures can be recommended in screening for MetS in routine clinical practice in order to combat CVD and type 2 diabetes [36]. In addition, this study was based on an adult population of health check-up. This limits the generalizability of the data to other populations to some extent. Further studies using the general population would be desirable.

\section{Conclusions}

The present study assessed longitudinal association between TG and other MetS components for the first time. The longitudinal association between TG and other MetS components increased with time. Among five pairs of TG and other MetS components, the correlation between TG and BMI was the largest. The correlation between TG and FPG was the smallest for men, while the correlation between TG and DBP was the smallest for women. It is important to closely monitor subjects with high levels of TG and BMI in health check-up population especially for women, because these two components are closely associated with development of hypertension, diabetes, CVD, and other chronic diseases.

\section{Acknowledgment}

The study is funded by National Natural Science Foundation of China (Serial Number: 81530087, $81502886,81373099)$, Young core personal project \& Beijing outstanding talent training project (Serial Number: 2014000020124G150), and Key Projects in the National Science \& Technology Pillar Program in the Twelfth Five-year Plan Period of China (Serial Number: 2014ZX10004005-001). We also would like to thank the medical personnel who participated in the data collection in the physical check-up department of Beijing Tongren Hospital and Beijing Xiaotangshan Hospital in China.

\section{Competing Interests}

The authors have declared that no competing interest exists.

\section{References}

1. Alberti KG, Eckel RH, Grundy SM, et al. Harmonizing the metabolic syndrome: a joint interim statement of the International Diabetes Federation Task Force on Epidemiology and Prevention; National Heart, Lung, and Blood Institute; American Heart Association; World Heart Federation; International Atherosclerosis Society; and International Association for the Study of Obesity. Circulation. 2009; 120:1640-5.

2. Chen YC, Sun CA, Yang T, et al. Impact of metabolic syndrome components on incident stroke subtypes: a Chinese cohort study. J Hum Hypertens. 2014; 28:689-93.

3. Sung KC, Rhee EJ, Ryu S, et al. Increased Cardiovascular Mortality in Subjects With Metabolic Syndrome Is Largely Attributable to Diabetes and Hypertension in 159971 Korean Adults. J Clin Endocrinol Metab. 2015; 100:2606-12. 
4. Eckel RH, Alberti KG, Grundy SM, et al. The metabolic syndrome. Lancet. 2010; 375:181-3.

5. Udell JA, Steg PG, Scirica BM, et al. Metabolic syndrome, diabetes mellitus, or both and cardiovascular risk in outpatients with or at risk for atherothrombosis. Eur J Prev Cardiol. 2014; 21:1531-40.

6. Bhowmik B, Afsana F, Siddiquee T, et al. Comparison of the prevalence of metabolic syndrome and its association with diabetes and cardiovascular disease in the rural population of Bangladesh using the modified National Cholesterol Education Program Expert Panel Adult Treatment Panel III and International Diabetes Federation definitions. J Diabetes Investig. 2015; 6:280-8.

7. Vonbank A, Saely $\mathrm{CH}$, Rein $\mathrm{P}$, et al. Insulin resistance is significantly associated with the metabolic syndrome, but not with sonographically proven peripheral arterial disease. Cardiovasc Diabetol. 2013; 12:106.

8. Sung $\mathrm{KC}$, Seo MH, Rhee EJ, et al. Elevated fasting insulin predicts the future incidence of metabolic syndrome: a 5-year follow-up study. Cardiovasc Diabetol. 2011; 10:108.

9. Ayubi E, Khalili D, Delpisheh A, et al. Factor analysis of metabolic syndrome components and predicting type 2 diabetes: Results of 10-year follow-up in a Middle Eastern population. J Diabetes. 2015; 7:830-8.

10. Seo MH, Bae JC, Park SE, et al. Association of lipid and lipoprotein profiles with future development of type 2 diabetes in nondiabetic Korean subjects: a 4-year retrospective, longitudinal study. J Clin Endocrinol Metab. 2011; 96:E2050-4

11. Kim SJ, Park YG, Kim JH, et al. Plasma fasting and nonfasting triglycerides and high-density lipoprotein cholesterol in atherosclerotic stroke: different profiles according to low-density lipoprotein cholesterol. Atherosclerosis. 2012; 223:463-7.

12. Kim EH, Lee JB, Kim SH, et al. Serum Triglyceride Levels and Cardiovascular Disease Events in Koreans. Cardiology. 2015; 131:228-35.

13. Stauffer ME, Weisenfluh L, Morrison A. Association between triglycerides and cardiovascular events in primary populations: a meta-regression analysis and synthesis of evidence. Vasc Health Risk Manag. 2013; 9:671-80.

14. Liu J, Zeng FF, Liu ZM, et al. Effects of blood triglycerides on cardiovascular and all-cause mortality: a systematic review and meta-analysis of 61 prospective studies. Lipids Health Dis. 2013; 12:159.

15. Akbulut G, Koksal E, Bilici S, et al. Metabolic syndrome (MS) in elderly: a cross sectional survey. Arch Gerontol Geriatr. 2011; 53:e263-6.

16. Yamamoto K, Okazaki A, Ohmori S. The relationship between psychosocial stress, age, BMI, CRP, lifestyle, and the metabolic syndrome in apparently healthy subjects. J Physiol Anthropol. 2011; 30:15-22.

17. Ying $X$, Song $Z Y$, Zhao CJ, et al. Body mass index, waist circumference, and cardiometabolic risk factors in young and middle-aged Chinese women. J Zhejiang Univ Sci B. 2010; 11:639-46.

18. Shamai L, Lurix E, Shen M, et al. Association of body mass index and lipid profiles: evaluation of a broad spectrum of body mass index patients including the morbidly obese. Obes Surg. 2011; 21:42-7.

19. Chen $\mathrm{C}, \mathrm{Lu}$ FC. The guidelines for prevention and control of overweight and obesity in Chinese adults. Biomed Environ Sci. 2004; 17 Suppl:1-36.

20. Schafer JL. Multiple imputation: a primer. Stat Methods Med Res. 1999; 8:3-15.

21. Andrinopoulou ER, Rizopoulos D, Geleijnse ML, et al. Dynamic prediction of outcome for patients with severe aortic stenosis: application of joint models for longitudinal and time-to-event data. BMC Cardiovasc Disord. 2015; 15:28.

22. Asar O, Ritchie J, Kalra PA, et al. Joint modelling of repeated measurement and time-to-event data: an introductory tutorial. Int J Epidemiol. 2015; 44:334-44.

23. Fieuws S, Verbeke G. Joint modelling of multivariate longitudinal profiles: pitfalls of the random-effects approach. Stat Med. 2004; 23:3093-104.

24. Chen F, Wang Y, Shan X, et al. Association between childhood obesity and metabolic syndrome: evidence from a large sample of Chinese children and adolescents. PLoS One. 2012; 7:e47380.

25. American Diabetes Association. Standards of medical care in diabetes-2015 abridged for primary care providers. Clin Diabetes. 2015; 33:97-111.

26. Johns BR, Abbasi F, Reaven GM. Estimates of insulin secretory function in apparently healthy volunteers vary as a function of how the relevant variables are quantified. Clin Chem. 2011; 57:627-32.

27. Zheng S, Zhou H, Han T, et al. Clinical characteristics and beta cell function in Chinese patients with newly diagnosed type 2 diabetes mellitus with different levels of serum triglyceride. BMC Endocr Disord. 2015; 15:21.

28. Hsiao FC, Hsieh $\mathrm{CH}, \mathrm{Wu} \mathrm{CZ}$, et al. Elevated fasting glucose levels within normal range are associated with an increased risk of metabolic syndrome in older women. Eur J Intern Med. 2013; 24:425-9.

29. Dayspring TD, Pokrywka G. Impact of triglycerides on lipid and lipoprotein biology in women. Gend Med. 2010; 7:189-205

30. Tenenbaum A, Klempfner R, Fisman EZ. Hypertriglyceridemia: a too long unfairly neglected major cardiovascular risk factor. Cardiovasc Diabetol. 2014; 13:159.

31. Guerin M, Le Goff W, Lassel TS, et al. Atherogenic role of elevated CE transfer from HDL to VLDL(1) and dense LDL in type 2 diabetes : impact of the degree of triglyceridemia. Arterioscler Thromb Vasc Biol. 2001; 21:282-8.

32. Saidu H, Karaye KM, Okeahialam BN. Plasma lipid profile in Nigerians with high--normal blood pressure. BMC Res Notes. 2014; 7:930.

33. Freiberg MS, Pencina MJ, D'Agostino RB, et al. BMI vs. waist circumference for identifying vascular risk. Obesity (Silver Spring, Md). 2008; 16:463-9.
34. Abbasi F, Blasey C, Reaven GM. Cardiometabolic risk factors and obesity: does it matter whether BMI or waist circumference is the index of obesity? Am J Clin Nutr. 2013; 98:637-40.

35. Qi L, Ding X, Tang W, et al. Prevalence and Risk Factors Associated with Dyslipidemia in Chongqing, China. Inter J Env Res Pub Heal. 2015; 12:13455-65.

36. Cheong KC, Ghazali SM, Hock LK, et al. The discriminative ability of waist circumference, body mass index and waist-to-hip ratio in identifying metabolic syndrome: Variations by age, sex and race. Diabetes Metab Syndr. $2015 ; 9 \cdot 74-8$ 